\title{
Universal Logic in terms of Quantum Information
}

\author{
Vasil Penchev, vasildinev@gmail.com \\ Bulgarian Academy of Sciences: Institute of Philosophy and Sociology: \\ Dept. og Logic and Philosophy of Science
}

\begin{abstract}
Any logic is represented as a certain collection of well-orderings admitting or not some algebraic structure such as a generalized lattice. Then universal logic should refer to the class of all subclasses of all well-orderings. One can construct a mapping between Hilbert space and the class of all logics. Thus there exists a correspondence between universal logic and the world if the latter is considered a collection of wave functions, as which the points in Hilbert space can be interpreted. The correspondence can be further extended to the foundation of mathematics by set theory and arithmetic, and thus to all mathematics.
\end{abstract}

Key words: bit and choice, Hilbert space, information, quantum information, qubit

\section{The thesis is:}

One can construct a mapping between Hilbert space and the class of all logics if the latter is defined as the set of all well-orderings of some relevant set (or class). That mapping can be further interpreted as a mapping of all states of all quantum systems, on the one hand, and all logics, on the other hand. The collection of all states of all quantum systems is equivalent to the world (the universe) as a whole. Thus that mapping establishes a fundamentally philosophical correspondence between the physical world and universal logic by the meditation of a special and fundamental structure, that of Hilbert space, and therefore, between quantum mechanics and logic by mathematics. Hilbert space can be interpreted as the free variable of "quantum information" and any point in it, as a value of the same variable as "bound" already. Furthermore, Hilbert space can be considered as that generalization of arithmetic, which is able to ground all mathematics possibly in a complete and consistent way, if the positive integers are substituted by the corresponding qubits constituting Hilbert space. This implies a one-to-one mapping between the transfinite ordinal numbers and the points of Hilbert space. Thus the correspondence between the physical world and universal logic can be extended to set theory and arithmetic founding mathematics in a kind of neo-Phytagoreanism.

\section{A short comment of the thesis and its history:}

Hegel is who introduced logic as ubiquitous and omnipresent and even as the synonym of ontology. Unfortunately, he did this in an unclear, complicated, inconsistent, intuitive and even mystical way. He developed a special kind of "logic of contradictions" called "dialectic logic" by him and opposed to the usual and traditional understanding of logic, which he designated as "formal logic". In addition, the so-called dialectic logic was compromised and discredited as an important part of the totalitarian state ideology of "marxism-leninism".

Hegel's idea can be considered as a form of neo-Pythagoreanism where the ontological position of number is replaced with a "dialectic and logical structure": "thesis - antithesis - synthesis".

Neither science, nor society corresponded to Hegel's idea. Dialectic logic could not be founded in a rigorous and mathematical, or properly logical way. Even more, Hegel opposed logic to dialectic logic to ground the latter ostensibly "logically". Thus dialectic logic was misunderstood being incorrectly founded and ahead of its time. 
Quantum mechanics was forced to describe uniformly discrete and smooth motions because the quantity of physical action is discrete for the Planck constant. A few fundamental scientific principles turn out to be prejudice and restricted to particular cases. "Quantum logics" appeared as the traditional Aristotelian logic of propositions and the corresponding Boolean lattice could also considered as an essential part of that prejudice of scientific common sense.

The concept of information introduced in different ways by Shannon (1948) and Kolmogorov (1965; 1968) being mathematically isomorphic to the thermodynamic quantity of entropy transfers bridge between artificial technical systems and natural processes: A new science appeared: “cybernetics” (Wienner, 1948).

The conception of quantum information was introduced in the theory of quantum information studying the phenomena of entanglement in quantum mechanics. The entanglement was theoretically forecast in the famous papers of Einstein, Podolsky, and Rosen (1935) and independently by Shrödinger (1935) deducing it from Hilbert space, the basic mathematical formalism of quantum mechanics. However, the former three demonstrated the forecast phenomenon as the proof of the alleged incompleteness of quantum mechanics. John Bell (1964) deduced a sufficient condition as an experimentally verifiable criterion in order to distinguish classical from quantum correlation (entanglement). Aspect, Grangier, and Roger (1981; 1982) confirmed experimentally the existence of quantum correlations exceeding the upper limit of the possible classical correlations. The theory of quantum information has thrived since the end of the last century in the areas of quantum computer, quantum communication, and quantum cryptography.

The thesis suggests that logic should be understood as some class of well-orderings. Some subclasses of that class admit but do not require some algebraic structures such as generalized lattices and some corresponding logical calculus. The same viewpoint includes all logics, or the conception of universal logic, in a natural way. Any logic of anything can be seen as a partial ordering and thus as a stage of the universal and single well-ordering of the universe going to the past. Any logic should be a partial result in the ordering in the course of time from future to past by the meditation of the choices made in the present.

Mathematics is the "language of nature" according to a metaphor become already traditional. The work suggests a literal interpretation of the metaphor. In turn and particularly, this implies a linguistic and semiotic approach to the foundation of mathematics. The set of semiotic triples (signifiers, signified, and signs) corresponds immediately to the mathematical concept of mapping (values, domain, and function) appealing just for it to be the ground of mathematics rather than that of set* Then "set" is any identity as a mapping, i.e. the particular case where "signifier" and "signified" coincide if one utilizes the semiotic terms. Furthermore, a large class of well-orderings satisfies the Dedekind-Peano axioms (Dedekind 1888; Skolem 1955; Germansky 1961) therefore allowing of arithmetic to be restored in the same ground.

\section{A few preliminary definitions and results:}

(I) The course of time can be described in terms of quantum mechanics as follows: The absolutely coherent state of the future de-coheres gradually into less and less entangled quantum systems by means of choices (or measurements) made in the present.

(II) Universal logic can be considered as the series of partial orderings of some universal class, e.g. that of all sets. Then, any given logic will be exactly one member of that series and can be defined:

(II.1) By the set, to which the partial orderings refer, and

\footnotetext{
* In fact, category theory realizes implicitly an analogical approach by the concepts both of functor and the dualities of functors and categories (Kuznetsov and Shvarts 1968).
} 
(II.2) By the rule, which can generate just the partial ordering, i.e. by the property, which describes the set of all well-orderings representing the partial ordering in question.

The definition (1) determines the logic as the logic of something where that something is the set, which has to be ordered and its logic means the way and degree of the ordering. The definition (1) includes both:

(II.1.a) Any scientific theory as the logic of the object of the theory, and properly

(II.1.b) The logics of something where that something is some set more interesting by the rule (2), which can generate it, rather than by itself.

The definition (2) includes both:

(II. 2.a) The case of the explicit property generating (all) well-orderings of any set independently of the interpretation of its elements, and

(II.2.b) The topological representation of the logic as the description of all well-orderings one by one: rather than as a common property determining unambiguously all well-orderings.

(III) The conception of quantum information allows of a natural ontological interpretation of universal logic:

There is a natural process of ordering in the course of time independent of what is ordered. What is ordered can be e.g. the world, i.e. the universe as a whole, or any part of it, i.e. any quantum system. So, universal logic can be interpreted as the successive partial results in the process of ordering independent of what is ordered. That ordering is a well-ordering and it originates from the course of time.

(IV) The unit of quantum information is the quantum bit, qubit definable as the normed superposition of any two orthogonal subspaces of the complex Hilbert space. Thus the complex Hilbert space underlying quantum mechanics is representable as the quantity of quantum information and any wave function, i.e. any state of any quantum system, being a point in it, can be seen as a value of that quantity. Consequently, all physical processes turn out to be quantum-informational, and nature or the universe is a quantum computer processing quantum information.

(V) Quantum information is equivalent to the generalization of information from finite to infinite series. The statement is trivial in the range of the axiom of choice.

(VI) Indeed the theorems about the absence of hidden variables in quantum mechanics (Neumann 1932; Kochen, Specker 1968) demonstrate that the mathematical formalism of quantum mechanics implies that no well-ordering of any coherent state might exist before measurement.

However, the same coherent state is transformed into a well-ordered series of results in time after measurement. In order to be equated the state before and after measurement, the well-ordering theorem equivalent to the axiom of choice is necessary.

The measurement mediating between them should be interpreted as an absolutely random choice of an element of the coherent state, for which no constructive way (equivalent to some hidden variable) can exist in principle.

Thus the quantity of quantum information can describe uniformly the state before and after measurement (equivalent to a choice among an infinite set).

\section{Direct arguments for the thesis:}

(A) The universal well-ordering in turn orders well all partial results, each of which is some logic. Consequently, the concept of universal logic means not only:

(A.1) A single universal and even logical mathematical order of all, but furthermore:

(A.2) A derivative, single, and universal order of all logics as a consecutive series of stages ordered into the single universal logic or order. 
(B) Consequently, the series of all logics turns out to be well-ordered. Even more, the quantity of quantum information can serve as a measure of "distance between two logics". The space of logics can be ordered in the distance of quantum information.

(C)The distance between any two logics can be measured by the quantity of quantum information. However any value of quantum information is a wave function: This means that a state of some quantum system corresponds to any two logics. Consequently, the state of anything in the sense of quantum mechanics is the distance of two orderings as logics of it.

(D) Any logic is unambiguously determined by both:

(D.1) The information distance from the coherent state (the absolute future), and

(D.2) The single well-ordering (the absolute past)

The orderings, which underlie the things for any two of the formers, represent a single state of the latter. Logic(s) is (are) identical to the world as a space of points and the same space as a vector one. That space is the complex Hilbert space underlying quantum mechanics and thus nature according to it. The space of all logics turns out to be identical and thus indistinguishable from the space of all things.

\section{An interpretation in terms of the foundation of mathematics}

A quantum information and semiotic approach to the foundation of mathematics can be defined. It originates from semiotic triples constituting mappings and from the concept of choice allowing of being chosen a signified for any signified and therefore a sign, and a mapping as a set of signs to be always constituted. Then the quantity of information representing the number of choices or signs turns out to be fundamental.

Set theory can be interpreted as a mathematical doctrine of infinity. However the quantities assigned to infinity such as ordinal and cardinal numbers are rather unspecified and not able to feature distinguishably an infinite set from another.

Hilbert space and all "points" in it can be assigned to infinity as a set of discerning "names" for all infinite sets. Indeed the complex Hilbert space can be seen as an immediate generalization of Peano's arithmetic as follows:

The $\mathrm{N}^{\text {th }}$ qubit can be interpreted as a "geometrical" generalization of the positive integer $\mathrm{N}$ after substituting the point "N" with the unit ball "N". Thus all values of this qubit "N" corresponds to the single point "N" after the "arithmetical degeneration" collapsing the qubit into a point.

Consequently, arithmetic and geometry turn out to be inherently linked in Hilbert space for it can be furthermore considered as an infinite-dimensional generalization of the usual Euclidean threedimensional geometry:

From the "viewpoint of arithmetic", the geometrical dimension of Hilbert space should refer to infinity describing exhaustedly any infinite set in a unique and relevant way. From the "viewpoint of geometry", the arithmetical dimension of Hilbert space is a degeneration from unit balls to points or the borderline case of a limit where the magnitude of the "unit" converges to zero. Both viewpoints can be unified into a single information one by the concept of choice as follows: The geometrical viewpoint is "before" the choice, and the arithmetic one, "after" it. Information and more precisely quantum information mean the quantity of those choices, each of which is a single, both geometrical and arithmetical unit.

Both arithmetical and geometrical dimension share infinity but in different ways in a sense: The arithmetical dimension implies rather the potential infinite of an infinite constructive process "adding and adding units, 1s, one by one": the "principle of (transfinite) induction". The geometrical dimension implies rather the actual infinite as the result of the above process as the limit of converging into a single point. 
The theorems about the absence of hidden variables in quantum mechanics (Neumann 1932; Kochen and Specker 1968) show not only that it is complete, but furthermore that Hilbert space, which is a sufficient premise for the theorems, implies that completeness by itself. One can say that the complex Hilbert space is inherently a complete mathematical structure. In fact, infinity in the set theory grounded on arithmetic remains a too undetermined concept accumulating contradictions without distinguishing discernibly an infinite set from any other of the same power. The concept of infinity though being exceptionally fruitful is what opens the "backdoor" of mathematics to contradictions. If the infinite sets are reliable differentiated from each other by their "wave function", this does not allow of identifying different infinities being due to the uncertainty of the term "infinity", and thus of sneaking contradictions through the holes of a fussy concept.

One can also complement that the completeness of Hilbert space, being inherently intrinsic, addresses some kind of neo-Pythagorean ontology.

\section{References}

Aspect, A., Grangier, R., Roger, G. (1981) Experimental Tests of Realistic Local Theories via Bells Theorem. Physical Review Letters, 47 (7): 460-463.

Aspect, A., Grangier, R., Roger, G. (1982) Experimental Realization of Einstein-Podolsky-RosenBohm Gedanken Experiment: A New Violation of Bells Inequalities. Physical Review Letters, 49 (2): 91-94.

Bell, J. (1964) On the Einstein Podolsky Rosen paradox. Physics (New York), 1 (3): 195-200.

Einstein, A., Podolsky, B., Rosen, N. (1935) Can Quantum-Mechanical Description of Physical Reality Be Considered Complete? Physical Review, 47(10): 777-780.

Dedekind, R. (1888) Was sind und was sollen die Zahlen? Braunschweig: Vieweg.

Germansky, B. (1961) The induction axiom and the axiom of choice. Mathematical Logic Quarterly, 7 (11-14), 219-223.

Kochen, S., Specker, E. (1968) The problem of hidden variables in quantum mechanics. Journal of Mathematics and Mechanics, 17(1): 59-87.

Kolmogorov, A. (1965) Tri podkhoda k opredeleniyu kolichestvo informatsii (Three approaches to the definition of the quantity of information). Probl. Inf. Transm., 1: 3-11.

Kolmogorov, A. (1968) Logical basis for information theory and probability theory. IEEE T. Inform. Theory, IT-14: 662-664.

Kuznetsov, V. V., Shvarts, A. S. (1968) Duality of functors and duality of categories. Siberian Mathematical Journal, 9 (4), 627-636.

Neumann, J. von (1932). Mathematische Grundlagen der Quanten- mechanik. Berlin: Springer, pp. 167-173 (Chapter IV.2).

Schrödinger, E (1935) Die gegenwärtige situation in der Quantenmechanik. Die

Naturwissenschaften, 23 (48), 807-812; 23 (49), 823-828, 23 (50), 844-849.

Skolem, T. (1955) Peano's axioms and models of arithmetic, in: Mathematical interpretation of formal systems (Studies in logic and the foundations of mathematics). Amsterdam: North-Holland Publishing Company, 1-14.

Shannon, C. (1948) A Mathematical Theory of Communication. Bell System Technical Journal, 27 (3), 379-423; 27 (4), 623-656.

Wiener, N. (1948) Cybernetics; or, Control and communication in the animal and the machine. Cambridge, Mass.: Technology Press. 